\title{
The clash between palliative care and COVID-19
}

\author{
Sebastiano Mercadante ${ }^{1}$ (I)
}

Received: 22 June 2020 / Accepted: 8 August 2020 / Published online: 14 August 2020

(C) Springer-Verlag GmbH Germany, part of Springer Nature 2020

Keywords Palliative care $\cdot$ COVID-19 $\cdot$ Hospice

After coronavirus disease 2019 (COVID-19) emerged in Wuhan, China, Italy was the first western country to be seriously affected by COVID-19 [1]. The other European countries have been involved with a certain delay. Escalating numbers of deaths have been reported elsewhere, particularly in USA and Brazil, and are expected to increase in many undeveloped countries. As a consequence, governments have adopted severe measures of social distancing. In Italy, a complete lockdown was announced on 9th of March and was discontinued on 3rd of May, when some activities were progressively allowed until the end of May, when almost all activities restarted. Social distancing is still recommended in closed environments, but uncontrollable events with numerous people are forbidden (for example, theaters, congresses, stadium). Other countries followed different policies in terms of timing and levels of lockdown. This dramatic social change was associated with incalculable direct and indirect economic damages and a collective psychological distress, rarely seen outside war time. However, as a consequence of the forced social distancing adopted from March to May, at the end of July in Italy, there was a flattened curve in the number of infected, healed, and deceased subjects, in comparison with other countries.

During this dramatic period, the prioritization of emergency care and critical care services produced a tremendous change of procedures, without the appropriate selection of care pathways, prognosis, and interdisciplinary evaluation. In an early COVID-19 phase, on the basis of the emotional wave, most serious patients were intubated for respiratory insufficiency, often followed by untrained staff, due to shortage of anesthesiologists and available beds, regardless of the unfavorable prognosis. Bad communication was of concern.

Sebastiano Mercadante

terapiadeldolore@lamaddalenanet.it; 03sebelle@gmail.com

1 Main Regional Center of Pain Relief and Supportive/Palliative Care, Hospice La Maddalena, Via San Lorenzo 312, 90146 Palermo, Italy
"We had to choice which patients to resuscitate and which not" was reported in the newspapers. Such declarations, emphasized by media, suggested that intensive care physicians wanted to overlook older or frail patients. Such populistic intent neglected what is widely shared by numerous scientific societies and recommendations on the need to avoid forms of therapeutic obstinacy, which often result in increasing the level of suffering of the dying patient, without the meaningful possibility of survival. Wrong decisions to hospitalize the majority of patients also increased the diffusion of contagion and did not help the hard work of physicians. While most patients infected with COVID-19 recovered, those who experienced severe illness and required hospitalization, intensive care, and intubation had escalating levels of mortality. The mortality rate of infected patients has been widely variable in the last months with numbers ranging from 5 to $0.7 \%$. This variability is likely to depend by testing rate in the population. Indeed, patients who required mechanical ventilation had very high mortality rates, estimated to be between 25 and $97 \%$. Mortality significantly increased in older patients with comorbidities. A lower mortality rate has been documented when using noninvasive ventilation [2].

\section{Integrating intensive care and palliative care}

Palliative care is a patient- and family-centered care with the aim of optimizing the quality of life by anticipating, preventing, and treating suffering, when "curative" therapies may be futile. Palliative care encompasses symptoms control and end-of-life issues, communication with caregivers, sets the goals of care ensuring dignity in death, and provides the appropriate decision-making process. However, effective application of palliative care in emergency units or intensive care requires specific knowledge and training, that anesthesiologists and critical care physicians often do not have [3]. Logistic issues such as protocols for patients' selection, application models, and triggers for consultation of external experts 
are still a matter of debate [4]. In the COVID-19 crisis, the early involvement of palliative physicians would have improved communication with caregivers, who in the meantime were completely excluded for the risk of contagion, and above all they could have helped any decision-making process for withdrawing, withholding, or intensification of the treatments. It is likely that with such an approach, many patients would have avoided dying in intensive care units after weeks of mechanical ventilation, despite no slightest chances of recovery. With an appropriate triage, only patients with the greatest chance of recovery should be eligible for life-sustaining treatment. Patients triaged not to receive intensive treatments require adequate relief of suffering and it is crucial that there is an alternative plan for supporting those patients outside this cohort. More relevantly, it is important to meet the needs of those outside the hospital system [5]. Governments should recognize the essential contribution of palliative care to the COVID-19 pandemic and ensure these services are integrated with the health care system response [6].

\section{Palliative care patients with COVID-19}

Some logistic problems emerged for palliative care patients with COVID-19. Strict visit policies and the risk of contagion made caring for patients dying with COVID-19 extremely difficult. Caregivers were troubled by the profound isolation during end-of-life care. Dying with dignity close to loved ones is a fundamental must for palliative care physicians. Caregivers felt a well-founded fear that their loved ones might die alone. For these reasons, some institutions provided "compassionate exceptions" to strict visit policies extending the provision of protective means to the visitors. Virtual visitation via an electronic device may be rapidly enabled across the healthcare system [7]. Continuous and compassionate communication between the palliative care team and patient's loved ones when the clinical condition changes is of paramount importance. Considering the unpredictable and rapid clinical changes that can happen with COVID-19, it is imperative that communication occurs at the time of admission at any level of care. All these competencies require large experience in communication which cannot be demanded to an unskilled staff. Given the level of complexity, health care professionals who are unfamiliar with palliative care should not be involved.

Some studies produced for rapid publication, mostly within 2 months of data collection, showed that cough, breathlessness, fatigue, and myalgia were the most significant symptoms in people hospitalized with COVID-19. In the dying patient, dyspnea was the most significant symptom. The cause of death was predominantly due to cardio-respiratory failure [8], rising the need for expertise in symptom control care of the dying patient [5].
Symptom burden, management, response to treatment, and outcomes were analyzed in 101 patients positive for COVID19 referred to hospital palliative care from March 4 to March 26, 2020. Older patients (range 72-89 years), presenting with comorbidities, and a lower Karnofsky level were most frequently referred. Referral to palliative care occurred a median of 4 days after hospital admission. The most frequent symptoms were breathlessness, agitation, drowsiness, pain, and delirium. Patients spent a median of 2 days under the palliative care team. As of March 30, 2020, about $3 / 4$ of patients had died (n.75), been discharged ( $n .13)$, or continued to receive palliative care input (n.13) [9].

A pandemic is a powerful amplifier of suffering, through physical and psychological stress, and through financial and social instability. Alleviation of suffering needs to be a key part of the response. Thus, the duty of palliative services is challenging in the response to COVID-19. Palliative care physicians have to be involved in triage, responding rapidly and flexibly, need to provide symptom management, organizing shifting resources into the community to facilitate psychosocial and bereavement care, also adopting measures to deal with stress, using technology to communicate with caregivers [10]. As COVID-19 presence is expected to persist for several months, even if in an attenuated way, hospice care could be relevant in the next future.

\section{Safeguarding palliative care for non-COVID-19 patients}

Regardless of the desirable involvement of palliative care physicians in patients with COVID-19, palliative care delivery care was extraordinarily affected by the pandemic in different ways. Worries about spreading and contracting COVID-19 have been growing in hospitals, possibly dissuading patients with symptoms from contacting palliative care services. The COVID-19 crisis has had an impact on the common pattern of admissions and discharges in palliative care units and hospices, in which visit restrictions were strictly applied according to government decision and health local policies, because of the fear of a possible contagion with caregiver admission. Unrequired recommendations reinforced these indications that are still valid in Italy in July. While patients admitted to acute palliative care units are mostly discharged home, hospice patients have a high mortality rate. Thus, caregivers often commented after hospice admission: "I will not see my loved one anymore." The consequence was a decrease in hospice admission during the lockdown period [11]. In the last months, caregivers were allowed to stay in the hospice room only after showing a negative pharyngeal swab (performed at their own expense, about 70 euros). While the decision to impede the visits for patients at the end of life is morally disputable, alternatives were objectively difficult to find. Of 
interest, in some areas in Italy, the risk of contagion was so small to do not justify such restrictions. In a large palliative care network in Sicily, including two home care programs, three hospices, and one acute palliative care unit, no patient or caregiver resulted to be COVID-19 positive. One hospice was closed to be eventually available for COVID-19 patients, but it has never been utilized.

Regretfully, psychological reactions of some physicians working in some areas strongly involved by pandemic showed frailty and burnout in such distressing circumstances. An article published in a national newspaper reported the sad aspects of the solitary death [12]. This article provoked measureless reactions on the socials, as it was considered a dangerous authoritative message that was equivocal for readers. Indeed, this criticism supported that the absence of caregivers could be compensated by the palliative care team. This interpretation is incomprehensible and misleading, possibly dictated by a "pallilalic" sense, a neologism indicating the palliative care omnipotence of some physicians. The solitary death was a fact, not an opinion. Palliative care may help render the last days of life more comfortable for patients and families. People may make "reflections" about new models and strategies to overcome such new situations, but none could be able to take the role of the spouse close to the patient. Information and directives from the health care system and scientific societies should be cautiously reported to prevent misinterpretations, given that palliative care is still not given a priority by local administrations. On the other side, palliative care teams have to make efforts for patients and their families without manifesting an excess of "pallilalia." To mitigate isolation during end of life, "compassionate exceptions" to strict visit policies and facilitating virtual visitation via electronic devices are recommended [13].

\section{Compliance with ethical standards}

Conflict of interest The author declares no conflict of interest.

\section{References}

1. Remuzzi A, Remuzzi G (2020) COVID-19 and Italy: what next? Lancet. 395(10231):1225-1228

2. Newport KB, Malhotra S, Widera E (2020) Prognostication and proactive planning in COVID-19. J Pain Symptom Manag;S0885-3924(20)30374-2

3. Cortegiani A, Russotto V, Raineri M et al (2018) Attitudes towards end-of-life issues in intensive care unit among Italian anesthesiologists: a nation-wide survey. Support Care Cancer 26:1773-1780

4. Mercadante S, Gregoretti C, Cortegiani A (2018) Palliative care in intensive care units: why, where, what, who, when, how. BMC Anesthesiol 18(1):106

5. Ferguson L, Barham D (2020) Palliative care pandemic pack: a specialist palliative care service response to planning the COVID19 pandemic J Pain Symptom Manag;S0885-3924(20)30177-9.

6. Fusi-Schmidhauser T, Preston NJ, Keller N, Gamondi C. (2020) Conservative management of COVID-19 patients-emergency palliative care in action. J Pain Symptom Manag :S08853924(20)30183-4

7. Makhoul A, Hoeksema L, Shoemaker L (2020) The role of palliative care in COVID-19. Cleve Clin J Med 2020

8. Keeley P, Buchanan D, , Carola C, et al. (2020) Symptom burden and clinical profile of COVID-19 deaths: a rapid systematic review and evidence summary BMJ Support Palliat Care;bmjspcare-2020002368

9. Lovell N, Maddocks M, Etkind SN, et al. (2020) Characteristics, symptom management, and outcomes of 101 patients with COVID19 referred for hospital palliative care. J Pain Symptom Manag. 2020;S0885-3924(20)30211-6

10. Etkind SN, Bone AE, Lovell N, et al. (2020) The role and response of palliative care and hospice services in epidemics and pandemics: a rapid review to inform practice during the COVID-19 pandemic $\mathrm{J}$ Pain Symptom Manage ;S0885-3924(20)30182-2.

11. Mercadante S, Giuliana F, Terruso L, Albegiani G (2020) The pattern of hospice pathways in the era of COVID-19. BMJ Supp Pall Care, in press

12. Mercadante S(2020) Perché è inaccettabile la morte solitaria. Repubblica

13. Mercadante $\mathrm{S}$, Adile C, Ferrera $\mathrm{P}$, Giuliana F, Terruso L, Piccione T (2020) Palliative care in the time of COVID-19. J Pain Symptom Manag 60:e79-e80

Publisher's note Springer Nature remains neutral with regard to jurisdictional claims in published maps and institutional affiliations. 